\title{
Variation in transpiration efficiency and its related traits in a groundnut (Arachis hypogaea L.) mapping population
}

\author{
L. Krishnamurthy ${ }^{\text {a }}$, V. Vadez ${ }^{\text {a,* }}$, M. Jyotsna Devi ${ }^{a}$, R. Serraj ${ }^{b}$, S.N. Nigam ${ }^{\text {a }}$, \\ M.S. Sheshshayee ${ }^{\mathrm{c}}$, S. Chandra ${ }^{\mathrm{a}}$, R. Aruna ${ }^{\mathrm{a}}$ \\ ${ }^{a}$ International Crops Research Institute for the Semi-Arid Tropics (ICRISAT), Patancheru 502324, Andhra Pradesh, India \\ ${ }^{\mathrm{b}}$ Crop \& Environmental Sciences Division, International Rice Research Institute (IRRI), DAPO Box 7777, Los Banos, Metro Manila, Philippines \\ ${ }^{\mathrm{c}}$ Department of Crop Physiology, University of Agricultural Sciences, Bangalore 560065, India \\ Received 23 April 2007; received in revised form 12 June 2007; accepted 12 June 2007
}

\begin{abstract}
Transpiration efficiency (TE) has been recognized as an important source of yield variation under drought stress in groundnut. Here the variation for TE is evaluated in a set of 318 recombinant inbred lines (RILs) of groundnut at $\mathrm{F}_{8}$ generation, derived from a cross between a high TE (ICGV 86031) and a low TE (TAG 24) parent, and the value of specific leaf area (SLA), SPAD chlorophyll meter readings (SCMR) and carbon isotope discrimination $\left(\Delta^{13} \mathrm{C}\right)$ as surrogates of TE are measured. Transpiration efficiency was measured gravimetrically in the 318 RILs and parents under progressive soil drying in a pot culture in two post-rainy seasons. Large and consistent variation for TE existed among the RILs across years. The overall distribution of TE among the RILs indicated that TE was governed by dominant and additive genes. Surrogates SLA and SCMR, were measured prior, during and after completion of the drought period, whereas $\Delta^{13} \mathrm{C}$ was measured on the dried tissue after harvest. Transpiration efficiency was negatively associated with SLA after the completion of stress treatment $\left(r^{2}=0.15\right)$ and $\Delta^{13} \mathrm{C}$ in leaves $\left(r^{2}=0.13\right)$ and positively associated with SCMR during stress $\left(r^{2}=0.17\right)$. These associations, all fairly weak, were significant only in 2004. None of these relationships was found in 2005. Although the heritability of SCMR during 2005 was relatively higher than that of TE, and although SCMR has previously been used to identify contrasting germplasm for TE, the stress-dependence of the relationship with TE, and the poor regression coefficients $\left(r^{2}\right)$ with that RIL population, do not confer that these surrogates are adequately robust enough in that population. Though more time consuming, a direct gravimetric evaluation for TE appeared to be more reliable.
\end{abstract}

(C) 2007 Elsevier B.V. All rights reserved.

Keywords: Transpiration efficiency; Groundnut; Arachis hypogaea L.; Drought stress; Specific leaf area; Carbon discrimination; SPAD chlorophyll meter reading

\section{Introduction}

Maximized use of stored soil water, increased biomass productivity per unit water use and highest conversion of vegetative biomass into economic yield are the ultimate goals of any drought research, according to a simple model of yield architecture (Passioura, 1977). Because groundnut is usually grown in rain fed conditions, it has been hypothesized that improving water use efficiency (WUE) would be the best strategy to cope with episodes of intermittent drought. Indeed, previous research intended to enhance the drought tolerance of groundnut has led to the selection of transpiration efficiency (TE) as an important component

\footnotetext{
* Corresponding author. Tel.: +91 40 30713463; fax: +91 4030713074.

E-mail address: v.vadez@cgiar.org (V. Vadez).
}

trait of WUE and as a major source of yield variation under drought stress (Nageswara Rao and Wright, 1994; Wright et al., 1994). In fact, WUE improvement has shown some success as a breeding objective in other crops like wheat (Condon et al., 2002; Rebetzke et al., 2002). A similar approach is currently being followed at ICRISAT where mapping populations involving contrasting parents for TE have been developed.

The growing need to find non destructive and less laborious methods of selection for improved TE, has subsequently led to the identification of surrogate traits that are closely related to TE such as specific leaf area (SLA) (Wright et al., 1994; Nageswara Rao and Wright, 1994), carbon isotope discrimination $\left(\Delta^{13} \mathrm{C}\right)$ (Hubick et al., 1986; Farquhar et al., 1988; Wright et al., 1994), SPAD Chlorophyll Meter Readings (Nageswara Rao et al., 2001; Bindu Madhava et al., 2003) and specific leaf nitrogen (SLN) (Nageswara Rao et al., 2001; 
Sheshshayee et al., 2006). The existence of a strong association between SLA and $\Delta^{13} \mathrm{C}$ as well as TE and a low genotype by environment interaction for the relationship between SLA and TE have led to the suggestion of SLA as an economical surrogate tool to select for TE (Wright et al., 1994). Also the subsequent findings of low SLA genotypes having greater photosynthetic capacity for unit leaf area (Nageswara Rao et al., 1995) further strengthened the suggestion of using leaf thickness (low SLA) as a selection criterion for enhancing TE in groundnut. In subsequent efforts to breed for drought tolerance in groundnut, low SLA, as a surrogate for high TE, was incorporated into genotypes with high transpiration and/or harvest index and selections were made from the same $\mathrm{F}_{2}$ base population either for low SLA (trait-based) or for pod yield (empirical) (Nigam et al., 2005). However, comparison of the progenies developed through trait-based and empirical breeding approaches, for enhanced pod yield of groundnut across environments, have shown that the trait-based selections did not demonstrate any clear superiority over the empirical selections for yield under drought (Nigam et al., 2003, 2005). This failure has necessitated re-examining the reasons for that, such as failure of the simple yield model to capture all the 'happenings' in the plant system, an imperfect selection index, negative associations among various yieldrelated physiological traits and failure of surrogate traits to explain the association between yield and its physiological components (Nigam et al., 2003). A particular reason for the unconvincing results of the trait-based approach is that genotypes with high TE often had low HI (Wright et al., 1994). This linkage has also prompted the need for the development of a component-trait approach using molecular marker tools, to characterize the parents precisely for TE, to identify relevant QTLs for TE that could be used in subsequent marker-assisted selection (Nigam et al., 2003, 2005). Recent studies conducted at ICRISAT, have indicated that two genotypes, ICGV 86031 and TAG 24, did vary for TE and also for its surrogates SLA and SCMR (Serraj et al., 2004a). Nautiyal et al. (2002) have also suggested the use of these two genotypes for combining high TE with high HI. A set of recombinant in-bred lines (RILs) were developed using ICGV 86031 and TAG 24 as two parents and advanced to $F_{8}$ stage for the purpose of genetic mapping.

The objectives of this study were: (i) to quantify the extent of genetic variation available for TE, measured gravimetrically, within this population, (ii) to investigate the consistency of TE measurement across seasons, and (iii) to study the effectiveness of SLA, SCMR and carbon isotope discrimination as proxies for TE for their potential future use in QTL mapping.

\section{Materials and methods}

\subsection{Management of experiments}

Recombinant inbred lines ( $\mathrm{F}_{8}$ generation) were developed, from a cross between ICGV 86031 and TAG 24, as single seed descendants from $\mathrm{F}_{3}$ generation (from $318 \mathrm{~F}_{3}$ plants descended from $12 \mathrm{~F}_{2}$ plants). The remaining $\mathrm{F}_{8}$ seeds after planting in 2004 were advanced to $F_{9}$ for use in 2005.

\subsubsection{4 season}

Pots of $23 \mathrm{~cm}$ diameter containing 8-kg of Alfisol mixed with $165 \mathrm{~g}$ of Multiplex ${ }^{\circledR}$ (vermi-compost) and $2 \mathrm{~g}$ of DAP were planted with a single seed of each RIL in a alpha-lattice $(16 \times 20$ blocks $)$ design in two separate sets. The first set, meant for harvest at the beginning of the drought stress imposition (pre-stress), was planted in three replications and the second, meant to be harvested after drought stress imposition (post-stress), with five replications. The top $3 \mathrm{~cm}$ surface soil was incorporated with $2 \mathrm{~g}$ of carbofuran ${ }^{\circledR}$ to avoid damages by soil-borne pests. The pots were inoculated with Rhizobium strain NC-29 (IC 7001) using liquid inoculation method (Brockwell, 1982) to ensure better nodulation. Seeds were sprayed with $2 \%$ Etherel solution and air-dried to break seed dormancy, if any. To manage data acquisition and harvest, the sowing and all subsequent activities were done in a staggered fashion as one replication per day starting 16 February 2004. All the pots were regularly optimally irrigated to $90 \%$ FC until 33 days after sowing. At 33 days after sowing (21 March 2004) the prestress set of plants were harvested to assess the leaf area, shoot and root biomass accumulated before the initiation of stress treatment. Soils of the post-stress set of pots were saturated with repeated watering on 20 March 2004, allowed to drain overnight, and the whole pots wrapped early in the morning with polythene bags to prevent soil evaporation. The pots were weighed as soon as bagged, and then left without irrigation till harvest. The pots were weighed subsequently, once in 5 days, to assess the level of water loss. Soil Plant Analytical Development (SPAD) Chlorophyll Meter Readings (SCMR) was recorded after bagging on all leaflets of the second most fully expanded leaf from the main stem, avoiding the mid-rib, and reading from each leaflet were averaged to give one SCMR value per replicated plant. Subsequent SCMR of the RILs were recorded on a new developed second most fully expanded leaf on the seventh day and on the tenth day (final harvest) after drought imposition. Plants were harvested when $80 \%$ of plants were showing symptoms of permanent wilting. Plant shoots were separated into leaves and stem and roots were washed to remove the soil. The leaves were retained in a moist polythene bag and the wrinkles gently heaved before measuring leaf area of the leaflets using Li COR area meter and the weights were recorded after drying the samples for at least $72 \mathrm{~h}$ in hot air ovens at $60{ }^{\circ} \mathrm{C}$.

\subsubsection{5 season}

There were only minor variations in the procedures adapted in this season compared to 2004. The soil used was $9 \mathrm{~kg}$ with $2.25 \mathrm{~g}$ DAP, and the randomization was changed to 4 rows $\times 80$ blocks. The pre-stress set also had five replications. The sowing started on 17 January 2005 and the drought stress imposition occurred 31 DAS (20 February 2005). In order to impose the 
stress more gradually, plants were allowed to lose no more than an average of $100 \mathrm{~g}$ of water per day, so that daily transpiration in excess of $100 \mathrm{~g}$ was added back to the pots. To do so, pots were weighted every 3 days, and water loss in excess of $300 \mathrm{~g}$ over 3 days was added back after weighing and calculating. This allowed a control over the kinetics of water stress imposition among plants that vary in size (leaf area), so that the decrease in soil moisture level followed roughly the same pattern in all plants. The plants were harvested replication by replication starting 20 March 2005 and ending 24 March 2005 in the same order of sowing, i.e. 28 days after sowing. An event of rain on the night between 8 and 9 March 2005 added variable amounts of water to the pots. The weighing of pots from all replications in the morning of 9 March allowed re-setting the pot reference weight for accurately measuring their transpiration in the forthcoming days. Additional water in the pots from the rain was then calculated as the difference between the pot weight on 9 March and an estimated pot weight on the evening of 8 March, based on estimates of transpiration on the days before the rain. Those transpiration estimates were assessed based on the plant-wise rate of decrease in transpiration in the previous 11-16 days, assuming a linear decrease in transpiration under water deficit, which is commonly the case (Ritchie, 1981; Sinclair and Ludlow, 1986).

\subsection{TE calculations}

In 2004, TE was calculated as follows, since no water was added back to the pots and water was only that found in the pot:

$\mathrm{TE}=\frac{\mathrm{DM}_{2}-\mathrm{DM}_{1}}{W_{2}-W_{1}}$

where $\mathrm{DM}_{1}=$ mean shoot biomass at pre-stress harvest; $\mathrm{DM}_{2}=$ shoot biomass at post-stress harvest; $W_{1}=$ weight of the pot after wrapping with polythene bag; $W_{2}=$ weight of the pot at the final harvest.

In 2005, TE was measured as follows:

$\mathrm{TE}=\frac{\mathrm{DM}_{2}-\mathrm{DM}_{1}}{\left(W_{2}-W_{1}\right)+\mathrm{WA}}$

where WA = water added to individual pots after regular weighing of the pots and compensating excess loss. WA includes the water addition from the rain.

The estimated dry weight increase during the experimental period, from transpiration and $\mathrm{TE}$ data in Table 1, was $4.4 \mathrm{~g} \mathrm{plant}^{-1}$ in 2004 and $8.8 \mathrm{~g} \mathrm{plant}^{-1}$ in 2005 . Assuming a ratio of dry to fresh weight of about $10 \%$, this would correspond to a fresh weight increase of 44 and 88 g plant $^{-1}$. This was negligible compare to the average cumulated transpiration over

Table 1

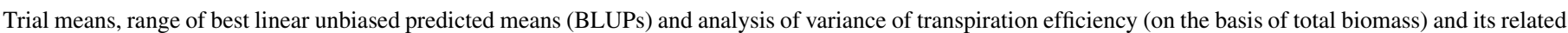
characteristics at stages related to stress imposition and harvest of 318 groundnut RILs and their parents TAG 24 and ICGV 86031 during 2004 and 2005

\begin{tabular}{|c|c|c|c|c|c|}
\hline Trait & Trial mean & Range of predicted means & S. Ed $( \pm)$ & $\sigma_{g}^{2}$ (S.E.) & Heritability $\left(h^{2}\right)^{\mathrm{a}}$ \\
\hline \multicolumn{6}{|c|}{ Transpiration (kg) } \\
\hline 2004 & 1.42 & $1.27-1.51$ & 0.051 & $0.002(0.0004)$ & 0.173 \\
\hline 2005 & 4.17 & $3.45-4.79$ & 0.212 & $0.080(0.0089)$ & 0.346 \\
\hline \multicolumn{6}{|c|}{ Transpiration efficiency $\left(\mathrm{g} \mathrm{kg}^{-1}\right)$} \\
\hline 2004 & 3.08 & $2.60-3.55$ & 0.282 & $0.066(0.011)$ & 0.182 \\
\hline 2005 & 2.14 & $1.92-2.36$ & 0.116 & $0.013(0.002)$ & 0.211 \\
\hline \multicolumn{6}{|c|}{ Specific leaf area $\left(\mathrm{cm}^{2} \mathrm{~g}^{-1}\right)$ at the start of stress } \\
\hline 2004 & 151.5 & $137.3-169.2$ & 7.57 & $51.4(7.9)$ & 0.299 \\
\hline 2005 & 177.3 & $155.6-201.9$ & 6.86 & $84.1(9.3)$ & 0.355 \\
\hline \multicolumn{6}{|c|}{ Specific leaf area $\left(\mathrm{cm}^{2} \mathrm{~g}^{-1}\right)$ at harvest } \\
\hline 2004 & 147.6 & $117.1-171.3$ & 9.28 & $101.4(12.3)$ & 0.287 \\
\hline 2005 & 144.5 & $126.1-163.5$ & 8.36 & $71.6(10.2)$ & 0.230 \\
\hline \multicolumn{6}{|c|}{ SCMR at the start of stress imposition } \\
\hline 2004 & 45.7 & $40.7-50.1$ & 2.14 & $5.0(0.64)$ & 0.256 \\
\hline 2005 & 44.3 & $39.1-50.4$ & 1.91 & $5.3(0.63)$ & 0.314 \\
\hline \multicolumn{6}{|c|}{ SCMR 5 days after stress imposition } \\
\hline 2005 & 41.0 & $35.0-46.0$ & 1.81 & $4.7(0.54)$ & 0.337 \\
\hline \multicolumn{6}{|c|}{ SCMR at 1 week after stress imposition } \\
\hline 2004 & 49.8 & $43.1-55.2$ & 2.59 & $6.7(0.92)$ & 0.232 \\
\hline \multicolumn{6}{|c|}{ SCMR 10 days after stress imposition } \\
\hline 2005 & 44.1 & $39.3-48.0$ & 1.53 & $3.8(0.41)$ & 0.381 \\
\hline \multicolumn{6}{|c|}{ SCMR 15 days after of stress imposition } \\
\hline 2005 & 45.8 & $39.8-50.9$ & 1.50 & $3.9(0.41)$ & 0.393 \\
\hline \multicolumn{6}{|c|}{ SCMR at harvest } \\
\hline 2004 & 49.7 & $42.9-55.8$ & 2.25 & $5.0(0.69)$ & 0.229 \\
\hline
\end{tabular}

${ }^{\text {a }}$ Heritability was estimated as $h^{2}=\sigma_{g}^{2} /\left(\sigma_{g}^{2}+\sigma_{e}^{2}\right)$. 
the experimental period of $1420 \mathrm{~g} \mathrm{plant}^{-1}$ in 2004 and $4170 \mathrm{~g} \mathrm{plant}^{-1}$ in 2005, and no adjustment to account for plant fresh mass increase in the " $W_{2}-W_{1}$ " component was considered necessary.

\subsection{Carbon isotope discrimination}

Leaf samples from the 2004 experiment were used to measure carbon isotope discrimination $\left(\Delta^{13} \mathrm{C}\right)$, which was calculated from measurements of the difference in carbon isotope ratios of the air and of the leaf samples. The sample analyzed was a bulk leaf sample from all the five replications. The ratio of the air was taken as $-7.6 \%$ on the PDB scale (Hubick et al., 1986). All leaves from final harvest samples after the exposure to drought stress were used. The dried material was ground to pass through a $100-\mu \mathrm{m}$ sieve. Isotope composition was measured by ratio mass spectrometry. Briefly, subsamples of approximately $10 \mathrm{mg}$ were combusted in an elemental analyzer (Carlo Erba Instrumazione, Italy). The combustion products were moved in a stream of helium, and $\mathrm{CO}_{2}$ in the effluent gas was separated from impurities chromatographically. $\mathrm{CO}_{2}$ was concentrated in a trap cooled with liquid $\mathrm{N}_{2}$ and the helium was pumped away. The trap was warmed and the $\mathrm{CO}_{2}$ was allowed to enter the inlet of the ratio mass spectrometer (VG SIRA 24) for measurement of isotope ratio. The isotope ratios of the samples were estimated by comparison with a working standard of $\mathrm{CO}_{2}$ with an isotope ratio of $-35.08 \%$ relative to $\mathrm{PDB}$.

\subsection{SLA measurements}

At all stages of the harvest the leaf area was measured using all the leaflets from each plant and the specific leaf area was estimated as $\mathrm{cm}^{2} \mathrm{~g}$ leaf dry weight.

\subsection{Statistical analysis}

The data from any individual experiment were analyzed using the following linear additive mixed effects model:

$Y_{i j k}=\mu+r_{i}+b_{i j}+g_{k}+e_{i j k}$

where $Y_{i j k}$ is the observation recorded on genotype $k$ in incomplete block $j$ of replicate $i, \mu$ the general mean, $r_{i}$ the effect of replicate $i, b$ the effect of block $j$ within replicate $i, g_{k}$ the effect of genotype $k$, and $e_{i j k}$ is the effect of the plot. The general mean $\mu$ and replicate effect $r_{i}$ were considered as fixed effects. The block effect $b_{i j}$, the RIL effect $g_{k}$, and the plot effect $e_{i j k}$, were assumed as random effects each with mean zero and constant variances $\sigma_{b}^{2}, \sigma_{g}^{2}$ and $\sigma_{e}^{2}$, respectively. Using the above model, the statistical procedure of residual maximum likelihood (ReML) method in GenStat statistical computing software was employed to obtain the unbiased estimates of the variance components $\sigma_{b}^{2}, \sigma_{g}^{2}$ and $\sigma_{e}^{2}$, and the best linear unbiased predictions (BLUPs) of the performance of the 318 RILs. Heritability was estimated as $h^{2}=\sigma_{g}^{2} /\left(\sigma_{g}^{2}+\sigma_{e}^{2}\right)$. The significance of genetic variability among RILs was assessed from the standard error of the estimate of genetic variance $\sigma_{g}^{2}$, assuming the ratio $\sigma_{g}^{2} /$ S.E. $\left(\sigma_{g}^{2}\right)$ to follow normal distribution asymptotically.

The above model was extended for over-year analysis of traits recorded in both years, assuming year effect as fixed, with genotype $\times$ year interaction (GEI) effect being a random effect assumed to have a mean of zero and constant variance $\sigma_{g E}^{2}$. The significance of GEI was assessed in a manner similar to that of $\sigma_{g}^{2}$. The significance of the fixed effect of the year was assessed using the Wald statistic that asymptotically follows a $\chi^{2}$ distribution and is akin to the $F$-test in the traditional ANOVA. All the statistical analyses were performed using GENSTAT (Version 8.1). Heritability across year was estimated as $h^{2}=\sigma_{g}^{2} /\left(\sigma_{g}^{2}+\sigma_{g E}^{2}+\sigma_{e}^{2}\right)$.

\section{Results}

The weather condition during the experimental periods is presented in Fig. 1. During the experimental period in 2004, the evaporative demand of the atmosphere was higher compared to 2005 as a consequence of higher maximum and minimum temperatures because the experiment was carried out roughly a month later. The rainy spell during 2005 that occurred almost half way through the stress imposition was critical as this decreased the VPD in those days after the rain.

\subsection{Differences in TE and surrogate traits}

There were significant differences among the RILs for total transpiration and TE in both the seasons (Table 1). A pooled analysis of both the experiments showed the existence of a
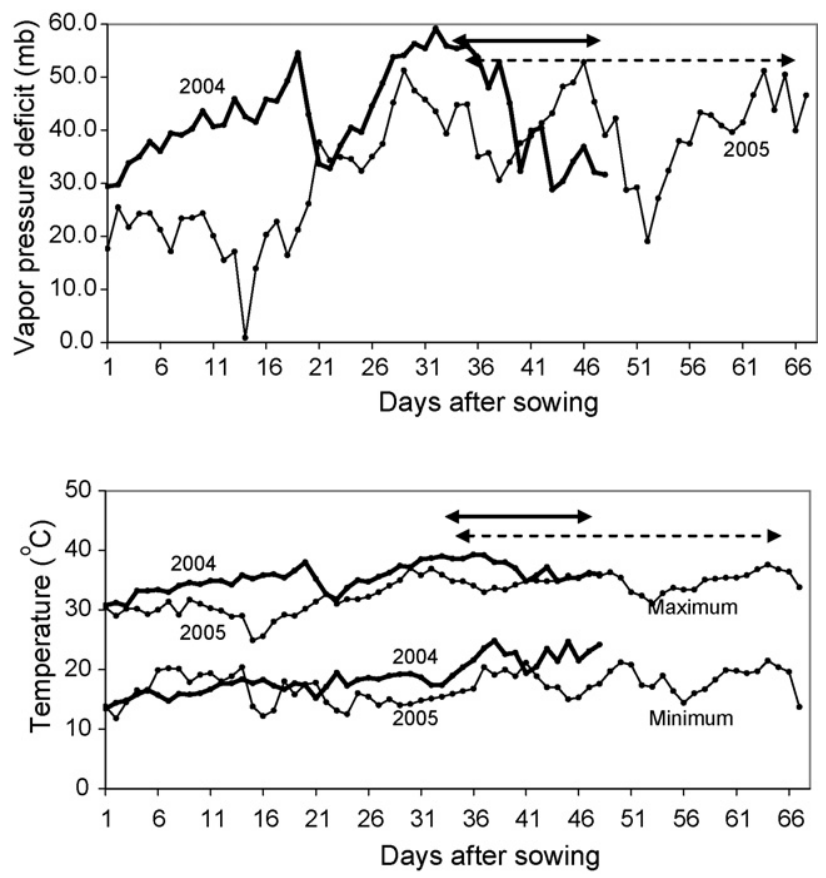

Fig. 1. Weather at the experimental site (ICRISAT, Patancheru) during the experimental period of 2004 (thicker lines) and 2005 (thinner lines). (Arrowheaded horizontal lines indicate the stress imposition period; solid $=2004$ and dashed $=2005$.) 


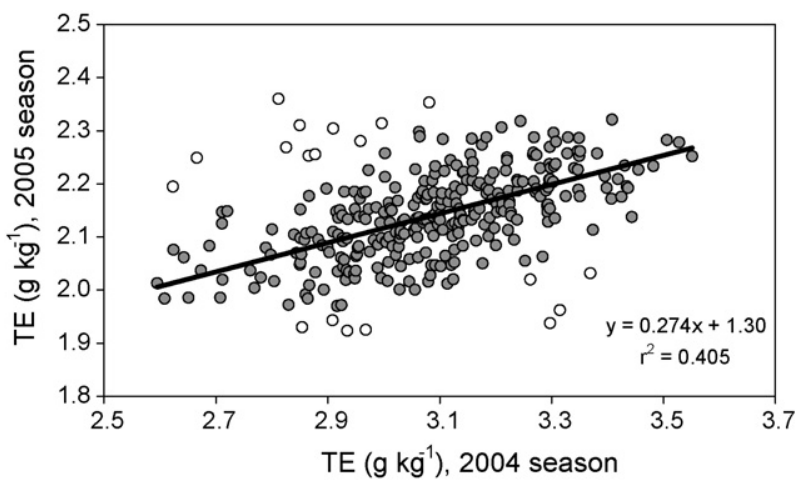

Fig. 2. Relationship between the TE $\left(\mathrm{g} \mathrm{kg}^{-1}\right)$ of $318 \mathrm{RILs}$ and their parents observed during 2004 and 2005. (The RILs, $n=19$, marked by open circles were not considered for the regression coefficient. See text for details.)

Table 2

Components of variance of transpiration efficiency ( $\mathrm{g} \mathrm{kg}^{-1}$ water) of the 318 RILs and their parents TAG 24 and ICGV 86031 across 2004 and 2005 experiments

\begin{tabular}{lllll}
\hline Fixed term & Wald statistic & d.f. & Wald/d.f. & Chi-sq. probability \\
\hline Experiments (E) & 1605.3 & 1 & 1605.3 & $<0.001$ \\
& & & & \\
Random term & & Component & S.E. \\
\hline RIL (R) & & 0.0313 & 0.0046 \\
$($ E) $\times(\mathrm{R})$ & 0.0006 & 0.0035 \\
Residual & & 0.183 & 0.0058 \\
\hline
\end{tabular}

significant RIL $\times$ season interaction for both transpiration $(0.0346 \pm 0.0041)$ and TE $(0.0140 \pm 0.0041)$. This interaction for TE was found to be a crossover type involving 19 RILs (Fig. 2). In an analysis without these 19 RILs, the RIL $\times$ season interaction was not significant $(0.0006 \pm 0.0035$; Table 2$)$, and these 19 RILs were considered as outliers. This interaction was likely due to the differences in vigor and health of seeds between the two seasons brought about by the seed size of a RIL used. During the first season, the limitation in seed number provided no option to be selective and almost all the seeds of a RIL had to be used for planting whereas during the second season the available seed supply was larger and provided for unconscious selection for larger seeds. This consequently would have influenced the plant vigor and health.

In spite of the difference in the range of TE observed in the RILs between the seasons, the performance of the RILs (plot of the BLUPs of 2004 and 2005 derived out of separate analysis) was close across the seasons (Fig. 2). The heritability of TE was close between the two experiments and was around 20\% (Table 1). Across year heritability value was $18 \%$. The distribution of the RILs for TE in both the experiments was monomodel-indicative of a polygenic control of this character with TAG 24 showing the lowest TE values and ICGV 86031 the above-average values (Fig. 3). Also large number of RILs performed better than the best parent, ICGV 86031, indicating that TE is governed more by dominant and additive genes. Some of the RILs that had consistently the highest TE during both the seasons were RIL 4, -5, -29, -56, -97, -98, -106, 116, 209 and -241 . The lines that had consistently the lowest TE were RIL $61,-75,-100,-121,-165,-207,-266,-282,-299$ and TAG 24.

SLA at the start of drought stress imposition as well as at harvest differed significantly among the RILs in both the seasons (Table 1). In a pooled analysis with both the seasons, the SLA of the RILs at the start of the stress imposition (no drought stress yet) differed significantly but there was no season $\times$ RIL interaction $(0.0 \pm 0.84)$ indicating that the relative differences among the RILs were the same across the seasons when no drought stress had occurred yet. On the contrary, the effects of RILs $(66.5 \pm 8.7)$ and the RIL $\times$ season $(20.5 \pm 6.1)$ were significant for the SLA at the final harvest after the drought stress. This indicated that the RIL performance in SLA at the final stage differed depending on the season. There was a general decline in SLA at the time of harvest compared to the SLA at the start of stress imposition and this decline was more pronounced during 2005 (Fig. 4). The
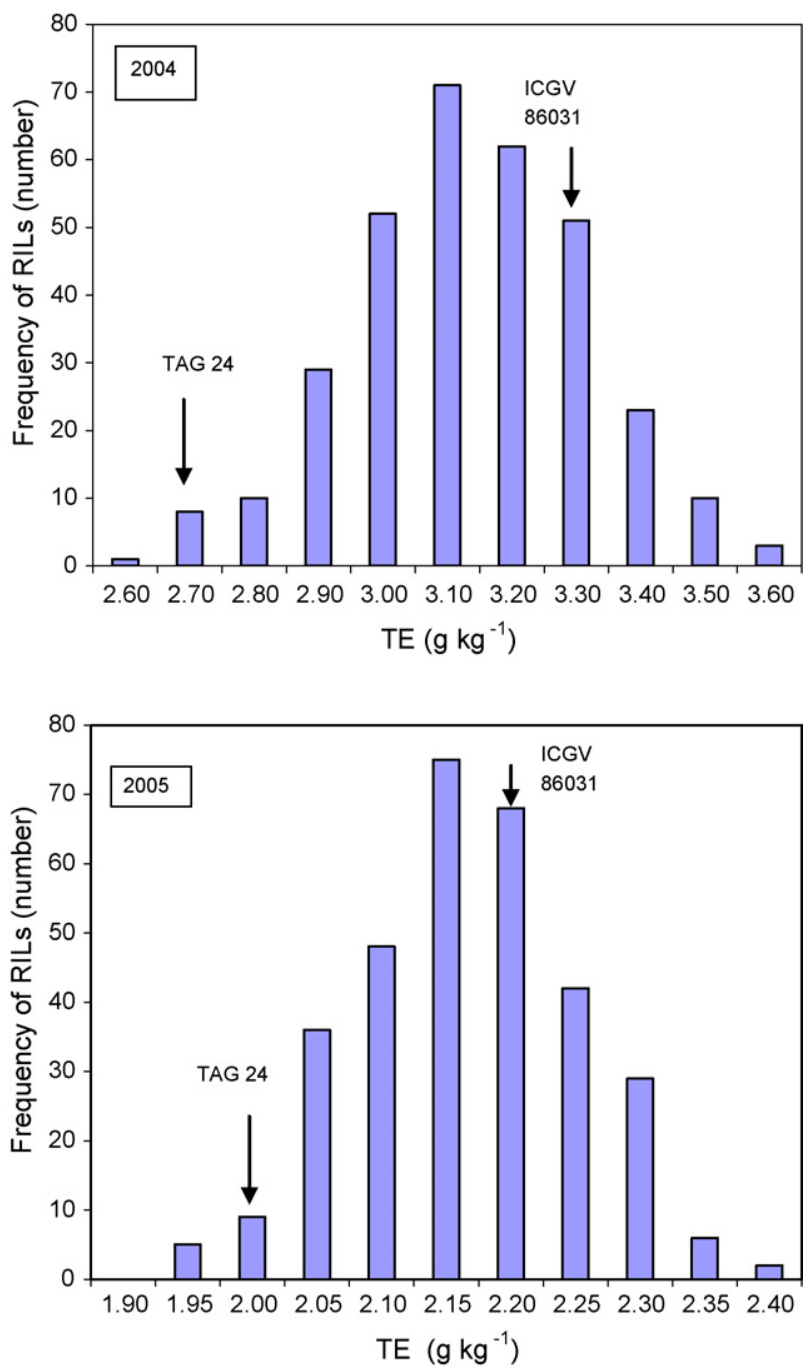

Fig. 3. Frequency distribution of transpiration efficiency $\left(\mathrm{g} \mathrm{kg}^{-1}\right)$ of 318 groundnut RILs along with their parents, ICGV 86031 and TAG 24, grown in a pot culture and exposed to progressively increasing drought stress till about 90\% loss of the available soil water fraction during 2004 and 2005. 

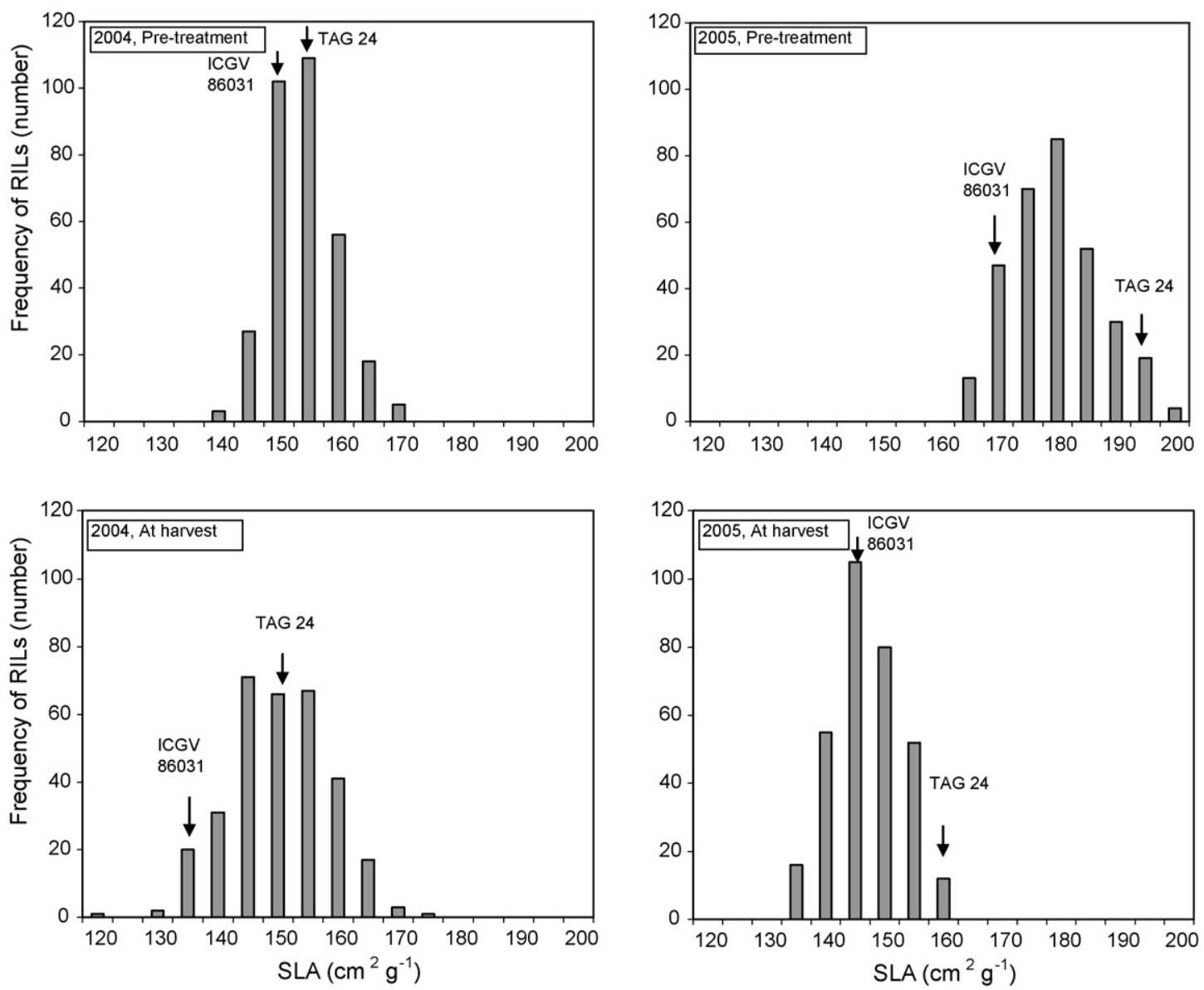

Fig. 4. Frequency distribution of SLA $\left(\mathrm{cm}^{2} \mathrm{~g}^{-1}\right)$ of 318 groundnut RILs along with their parents, ICGV 86031 and TAG 24, before and after (at harvest) drought stress imposition during 2004 and 2005.

range of SLA among the RILs observed after the stress imposition was closer between the seasons than was that of the SLA observed before the stress imposition. The heritability values of SLA were relatively larger before the stress imposition (0.30 in 2004 and 0.36 in 2005) than after the stress imposition (0.29 in 2004 and 0.23 in 2005).

SCMR at the start of stress imposition in both seasons, at 7 and 10 days after imposing the stress in 2004, and at 5, 10 and 15 days after imposing the stress in 2005 showed large and significant variation between RILs (Table 1). In a pooled analysis of both the seasons, the RIL $\times$ season effect was nonsignificant at the start of the stress imposition $(0.18 \pm 0.24)$ whereas this effect was highly significant at the final harvest in 2004 and 15 days after stress imposition in $2005(0.85 \pm 0.27)$ indicating that the RIL performance in SCMR was the same in both the years at the start of stress imposition when no drought stress had occurred yet, whereas at the final stage, after drought stress had occurred, the performance of the RILs differed depending on the season. The heritability values observed for the SCMR was the highest among all the traits studied, particularly during 2005. It is worth noticing that the SCMR values increased as the intensity of stress advanced during 2005 (Table 1).

Carbon isotope discrimination values of the RILs ranged from 18.0 to 20.3 and this range was higher than the range reported for eight diverse groundnut cultivars used (Wright et al., 1988).

\subsection{Relationship among TE, SLA, SCMR and $\triangle^{13} C$}

At the stress imposition stage, there was a significant correlation between the SLA values of 2004 and 2005 ( $r=0.64$; $p=<0.001)$. At this stage, there was no relationship between SLA and TE (2004: $r=-0.03$; 2005: $r=-0.15$ ). The relation between SLA values of 2004 and 2005 at harvest was also significant although much poorer $(r=0.44 ; p=<0.001)$, consistent with the above statement of a larger $\mathrm{G} \times \mathrm{E}$ effect on SLA at harvest than at the beginning of the stress. Yet, it was at that stage that TE correlated significantly and negatively with SLA during 2004 (Fig. 5) and during 2005. However, the closeness of the fit was generally poor, and even poorer in 2005 $(r=-0.19 ; p=0.05)$ than in $2004(r=-0.38 ; p=<0.001)$. In fact, the difference in SLA between the two parents was relatively low $\left(147 \mathrm{~cm}^{2} \mathrm{~g}^{-1}\right.$ DW in ICGV 86031 compared to $153 \mathrm{~cm}^{2} \mathrm{~g}^{-1}$ DW before drought stress imposition during 2004 and $165 \mathrm{~cm}^{2} \mathrm{~g}^{-1} \mathrm{DW}$ compared to $192 \mathrm{~cm}^{2} \mathrm{~g}^{-1} \mathrm{DW}$ during 2005.) compared to the range among the RILs (Fig. 4) indicating the occurrence of large transgressive segregation for SLA.

Similar to SLA, SCMR values in 2004 and 2005 at the stress imposition stage were significantly correlated $(r=0.63$; 

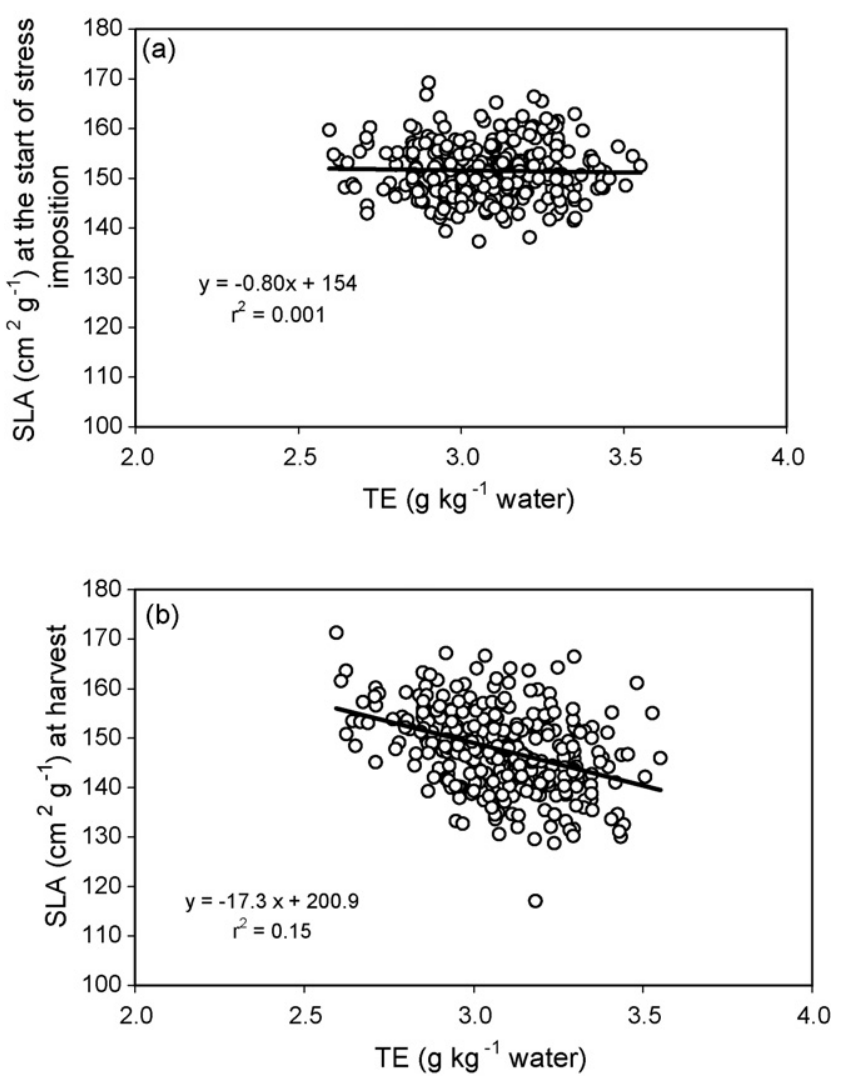

Fig. 5. Relationship between the TE $\left(\mathrm{g} \mathrm{kg}^{-1}\right)$ and the SLA $\left(\mathrm{cm}^{2} \mathrm{~g}^{-1}\right)$ (a) at the start of the stress imposition stage and (b) at harvest of 318 RILs and their parents observed during 2004.

$p=<0.001)$. Again, there was no relationship between SCMR and TE at this stage (2004: $r=-0.07 ; 2005: r=0.03$ ). The relationship between SCMR values observed 7 days after stress imposition in 2004 and those observed 10 days after stress imposition in 2005 was also significant $(r=0.43 ; p=<0.001)$, although again relatively weak, repeating the $\mathrm{G} \times \mathrm{E}$ effect that was seen for SLA at harvest. Then, SCMR was positively correlated with TE at 7 days after stress imposition in 2004 alone (Fig. 6). By contrast in 2005, SCMR at no stage showed any relationship with TE. As in the case of SLA, the differences in SCMR between the two parents were also relatively low across stages (2-6 units) compared to the range that could be seen among the RILs (9-13 units) indicating a similar transgressive segregation pattern for SCMR among the RILs, in a similar fashion to SLA values.

Transpiration efficiency exhibited a significant linear negative relationship with $\Delta^{13} \mathrm{C}\left(r^{2}=0.13 ; p=0.001\right.$, Fig. 7) in $2004\left(\Delta{ }^{13} \mathrm{C}\right.$ not measured in 2005). Comparatively, this relationship was as close to the relationships that were observed between TE and SLA at harvest and SCMR at 7 days after stress imposition during 2004.

\section{Discussion}

Overall, 2 years of phenotyping for TE in this RIL population showed a fairly good consistency in TE of RILs
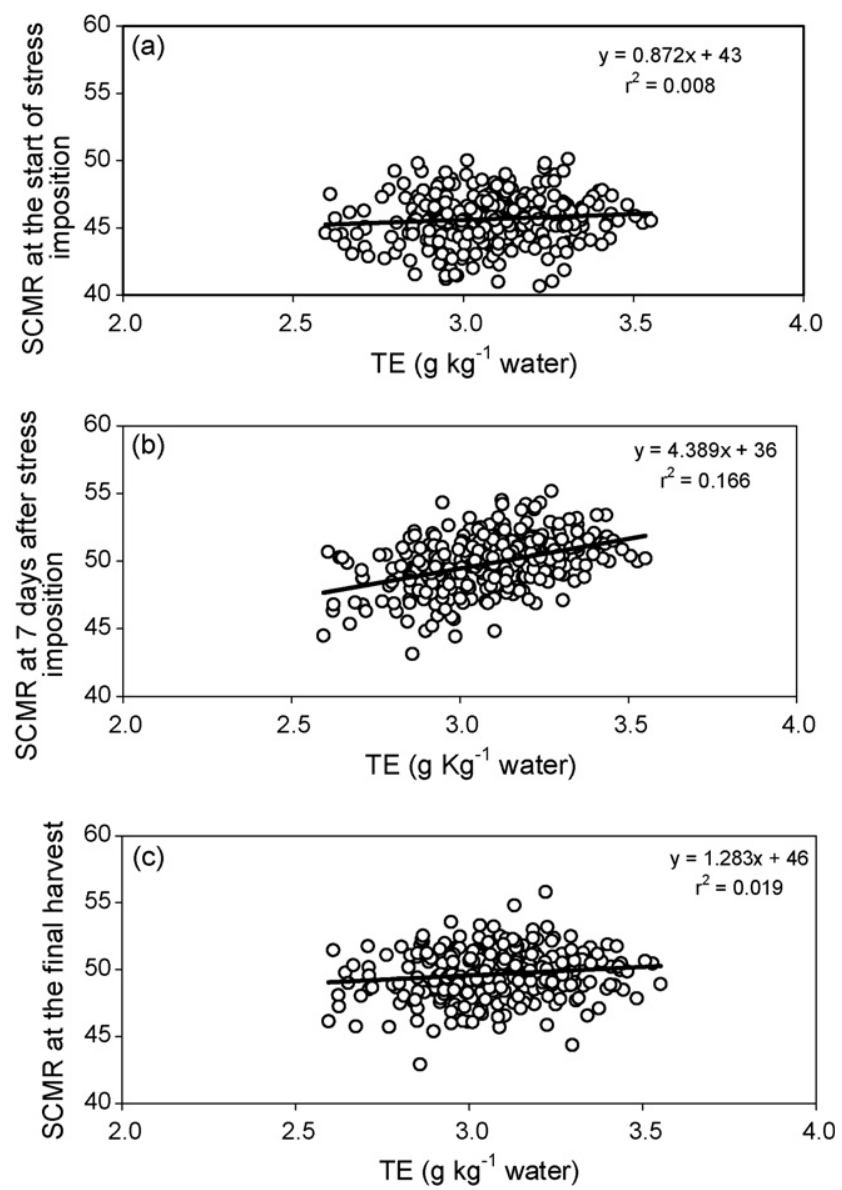

Fig. 6. Relationship between the TE $\left(\mathrm{g} \mathrm{kg}^{-1}\right)$ and the SCMR (a) at the start of the stress imposition stage, (b) at 7 days after the stress imposition and (c) at the final harvest (11 days after stress imposition) of 318 RILs and their parents observed during 2004.

across seasons/years/watering regimes/VPD, in spite of the range of variations among RILs being much lower in one season, and of the differences in evaporative demands in the two seasons. This probably shows the robustness of the trait across environments with negligible exceptions. This data also showed that the use of SLA and SCMR as surrogates for the

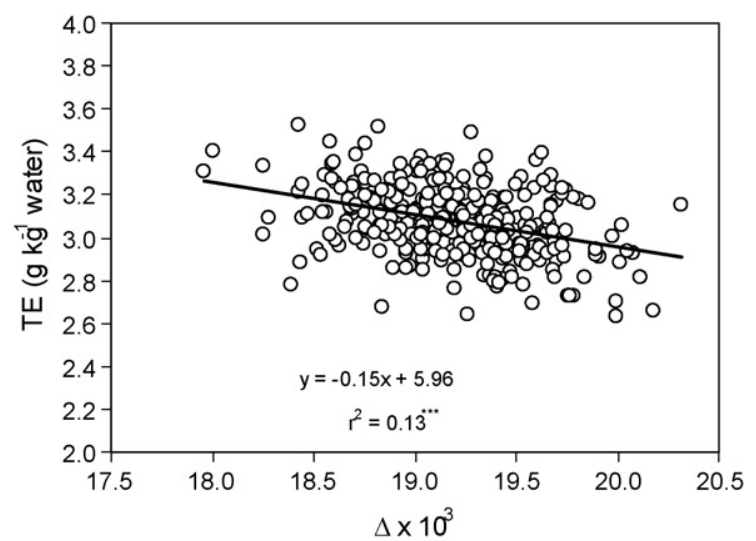

Fig. 7. Relationship between the $\mathrm{TE}\left(\mathrm{g} \mathrm{kg}^{-1}\right)$ and the carbon isotope discrimination at the final harvest after the stress imposition of 318 RILs and their parents observed during 2004. 
purpose of phenotyping for TE in this RIL population had to be considered carefully as the relationships were not adequately close and consistent across seasons.

The difference between the RILs with the lowest and the highest TE was 37\% during the first season and $23 \%$ during the second season. It might be speculated that the lower range in 2005 could be related to the fact that the water stress was more gentle because of: (i) the conditions of a lower evaporative demand since the experiment was carried out earlier in the season; (ii) the event of rain in 2005 further decreased the overall VPD of the experiment; (iii) plants were partially re-watered to imposed a gradual stress, whereas plants depleted the pot moisture with no partial re-watering in 2004, which imposed a relatively fast stress (about 10 days to reach the end). In fact, mean VPD values during the drought stress imposition time across seasons were 4.1 and $4.0 \mathrm{kPa}$ in 2004 and 2005. Therefore, both values were relatively high and it seems unlikely that these may have induced large differences in the response of the RIL at these levels of VPD. In any case, more work is needed to further investigate the $\mathrm{G} \times \mathrm{VPD}$ interaction for TE and to determine an optimum water stress treatment, both with the purpose of minimizing $G$ by $E$ effect on TE and of maximizing the range of variation for TE. In any case, a salient result from this work was that TE values were consistent across seasons in these experimental conditions.

This range of variation in TE was substantial enough for further selection and use in breeding programs for drought tolerance. This range is also suitable for QTL mapping of TE, which is being planned once sufficient number of polymorphic markers is available for that population. The range observed (2.60-3.55 and 1.92-2.36 $\mathrm{g} \mathrm{kg}^{-1}$ ) was in accordance with the WUE range recorded in a large number of groundnut genotypes (Wright et al., 1988, 1994; Nageswara Rao and Wright, 1994; Hebbar et al., 1994). There were seven RILs that gave TE values significantly higher than that of the best parent during 2004 and about 50 that were equal or higher than that of the best parent (ICGV 86031) during 2005. The range of TE variation observed within these RILs were expected to be the maximum as their parents (TAG 24 and ICGV 86031) were confirmed to have large contrast for TE (Serraj et al., 2004a) and were even specifically proposed earlier for use in developing improved drought-tolerant cultivars (Nautiyal et al., 2002).

The heritability value observed for $\mathrm{TE}$ in the individual experiments was close to $20 \%$ in this study and this was $18 \%$ across experiments. However, adding more reps to the experimental design would further enhance the heritability values. The heritability for TE has been shown to be low (34\%) compared to that of the carbon isotope discrimination (53\%) in a study using $44 \mathrm{~F}_{2}$ populations (Hubick et al., 1988). Also the distribution of the RILs for TE indicated a polygenic control of this character and involvement of dominant and additive genes as seen for shoot biomass in chickpea (Cicer arietinum L.) under terminal drought (Serraj et al., 2004b). Therefore, application of marker-assisted selection can result in better efficiency of selection for high TE, and efforts are currently in progress in this direction (Nigam et al., 2005).
In the present study, both SLA and SCMR values of RILs, recorded before the imposition of drought stress did not show any relationship with the TE at this stage, whereas SLA at the time of final harvest and the SCMR at 7 days after stress imposition in 2004 showed a significant relationship with TE. These data suggest that the trait SLA is adaptive in nature and water deficit has been shown to reduce SLA — part of an adaptive mechanism to reduce leaf area and transpiration (Wright et al., 1993; Brown and Byrd, 1996). Similar adaptive changes in SLA of eight groundnut genotypes were also reported in response to growing temperature (Craufurd et al., 1999). These data show that the surrogate should be used under specific situation only.

In any case, surrogate traits SLA and SCMR showed only occasional, poor and stress level-dependant association with TE. This is in contrast with several previous reports showing the existence of a close association of TE with SLA or SCMR. These works, however, involved a very limited number of genotypes (Wright et al., 1994; Nageswara Rao and Wright, 1994; Nageswara Rao et al., 2001; Bindu Madhava et al., 2003; Sheshshayee et al., 2006). The poor relationship between TE and surrogates in this study or in previous one (Nautiyal et al., 2002) is unclear. It may be related to the narrower range of variation for SLA and SCMR, than the variation for TE between the two genotypes involved in that cross. This narrow variation for SLA and SCMR between ICGV 86031 and TAG 24 is also reported by recent work where the variation among six highly contrasting genotypes for TE, SLA and SCMR was: 2.76$3.58 \mathrm{~g} \mathrm{~kg}^{-1}$ for TE, 222-293 $\mathrm{cm}^{2} \mathrm{~g}^{-1}$ for SLA and 33.0-41.0 units for SCMR, whereas the variations between ICGV 86031 and TAG 24 are still fairly high for TE (2.99-3.58 $\left.\mathrm{g} \mathrm{kg}^{-1}\right)$, but relatively limited for SLA $\left(222-250 \mathrm{~cm}^{2} \mathrm{~g}^{-1}\right)$ and for SCMR (37.7-39.0) (Sheshshayee et al., 2006). This would indicate that differences in TE between these genotypes are not closely linked to differences in SLA or SCMR between these, and would explain the lack of a close relation between SLA or SCMR and TE in their progenies. Therefore, the use of these surrogates may not be suitable in that population.

Similar conclusion could be taken from $\Delta^{13} \mathrm{C}$ data, although among peanut genotypes, the existence of a negative relationship of TE with $\Delta^{13} \mathrm{C}$ has been shown in a number of studies (Wright et al., 1988, 1994; Nageswara Rao and Wright, 1994; Craufurd et al., 1999; Bindu Madhava et al., 2003; Sheshshayee et al., 2006). In the present work, the poor relation between TE and $\Delta^{13} \mathrm{C}$ may be related to the fact that all the leaves were used to evaluate $\Delta^{13} \mathrm{C}$ and not only those that were produced during the experimental period. These two pools of leaves may not have similar $\Delta^{13} \mathrm{C}$ values and this may have distorted the relation. Yet, it raises concern about the use of $\Delta^{13} \mathrm{C}$ data for phenotyping purposes in molecular breeding programs.

\section{Conclusion}

Large, useful and consistent TE variation exists in this mapping population. Transpiration efficiency data were robust across years, and surrogate trait usually employed to proxy TE turned out to be little related to TE data in this work. Considering the low heritability of TE, it is suggested that efforts need to be 
developed to improve the precision of phenotyping for TE to achieve a higher heritability. Yet, given the relatively small range of variation for TE measured gravimetrically between the parental genotypes, efforts are probably needed to explore further the existing variability for TE in the groundnut germplasm, which has not been explored yet, and which may reveal much larger variation than that reported in these 2 parents. The potential surrogates like SLA, SCMR and carbon isotope discrimination were significantly associated with TE, although their relationship seemed to be limited to specific stages of stress development and the percentage of variation accounted in these relationships were too low to merit their use as indirect indicators of TE variations. Therefore, it seems necessary to rely more on direct evaluation for TE for the purpose of phenotyping until the relationships of TE with its "traditional" surrogates are robust enough to be useful. At ICRISAT we have been capable of performing that measurement. Though labor consuming, it is currently done on a fairly routine basis, and it has a potential of being largely automated.

\section{Acknowledgements}

The authors gratefully acknowledge the technical assistance provided in conducting the experiments and collection of data by the technicians of Crop Physiology Lab and Groundnut breeding, in particular Mr. M. Anjaiah and P. Arjun Rao, and the useful comments and suggestions given by Dr. F.R. Bidinger, Consultant Physiologist at the Crop Physiology Laboratory, to improve the manuscript. Authors are thankful to the Generation Challenge Program for their support to this study through funding of project \#7.

\section{References}

Bindu Madhava, H., Sheshshayee, M.S., Shankar, A.G., Prasad, T.G., Udayakumar, M., 2003. Use of SPAD chlorophyll meter to assess transpiration efficiency of peanut. In: Cruickshank, A.W., Rachaputi, N.C., Wright, G.C., Nigam, S.N. (Eds.), Breeding of Drought-Resistant Peanut. Proceedings of a Collaborative Review Meeting held at ICRISAT, Hyderabad, India, 25-27 February 2002. ACIAR Proceedings No. 112, Canberra. pp. 3-9.

Brockwell, J., 1982. Inoculation methods for field experimenters and farmers. In: Vincent, J.M. (Ed.), Nitrogen Fixation in Legumes. Academic Press, New York, pp. 211-221.

Brown, R.H., Byrd, G.T., 1996. Transpiration efficiency, specific leaf weight, and mineral concentration in peanut and pearl millet. Crop Sci. 36, 475-480.

Condon, A.J., Richards, R.A., Rebetzke, G.J., Farquhar, G.D., 2002. Improving intrinsic water use efficiency and crop yield. Crop Sci. 42, 122-131.

Craufurd, P.Q., Wheeler, T.R., Ellis, R.H., Summerfield, R.J., Williams, J.H., 1999. Effect of temperature and water deficit on water-use efficiency, carbon isotope discrimination, and specific leaf area in peanut. Crop Sci. 39, 136-142.

Farquhar, G.D., Hubick, K.T., Condon, A.G., Richards, R.A., 1988. Carbon isotope fractionation and plant water-use efficiency. In: Rundel, P.W., et al. (Eds.), Applications of Stable Isotope Ratios to Ecological Research. Springer-Verlag, New York, pp. 21-40.

Hebbar, K.B., Sashidhar, V.R., Udaykumar, M., Devendra, R., Nageswara Rao, R., 1994. A comparative assessment of water use efficiency in groundnut
(Arachis hypogaea) grown in containers and in the field under water-limited conditions. J. Agric. Sci. (Camb) 122, 429-434.

Hubick, K.T., Farquhar, G.D., Shorter, R., 1986. Correlation between water-use efficiency and carbon isotope discrimination in diverse peanut (Arachis) germplasm. Aust. J. Plant Physiol. 13, 803-816.

Hubick, K.T., Shorter, R., Farquhar, G.D., 1988. Heritability and genotype $\times$ environment interactions of carbon isotope discrimination and transpiration efficiency in peanut (Arachis hypogaea L.). Aust. J. Plant Physiol. $15,799-813$.

Nageswara Rao, R.C., Wright, G.C., 1994. Stability of the relationship between specific leaf area and carbon isotope discrimination across environments in peanut. Crop Sci. 34, 98-103.

Nageswara Rao, R.C., Udaykumar, M., Farquhar, G.D., Talwar, H.S., Prasad, T.G., 1995. Variation in carbon isotope discrimination and its relationship to specific leaf area and ribulose-1,5-bisphosphate carboxylase content in groundnut genotypes. Aust. J. Plant Physiol. 22, 545-551.

Nageswara Rao, R.C., Talwar, H.S., Wright, G.C., 2001. Rapid assessment of specific leaf area and leaf nitrogen in peanut (Arachis hypogaea L.) using a chlorophyll meter. J. Agron. Crop Sci. 186, 175-182.

Nautiyal, P.C., Rachaputi, N.R., Joshi, Y.C., 2002. Moisture-deficit induced changes in leaf-water content, leaf carbon exchange rate and biomass production in groundnut cultivars differing in specific leaf area. Field Crops Res. 74, 67-79.

Nigam, S.N., Cruickshank, A.W., Rachaputi, N.C., Wright, G.C., Basu, M.S., 2003. Where to from here? In: Cruickshank, A.W., Rachaputi, N.C., Wright, G.C., Nigam, S.N. (Eds.), Breeding of Drought-Resistant Peanut. Proceedings of a Collaborative Review Meeting held at ICRISAT, Hyderabad, India, 25-27 February 2002. ACIAR Proceedings No. 112, Canberra. pp. 91-92.

Nigam, S.N., Chandra, S., Rupa Sridevi, K., Bhukta, M., Reddy, A.G.S., Rachaputi, N.R., Wright, G.C., Reddy, P.V., Deshmukh, M.P., Mathur, R.K., Basu, M.S., Vasundhara, S., Vindhiya Varman, P., Nagda, A.K., 2005. Efficiency of physiological trait-based and empirical selection approaches for drought tolerance in groundnut. Ann. Appl. Biol. 146, 433-439.

Passioura, J.B., 1977. Grain yield, harvest index and water use of wheat. J. Aust. Inst. Agric. Sci. 43, 21.

Rebetzke, G.J., Condon, A.G., Richards, R.A., Farquhar, G.D., 2002. Selection for reduced carbon isotope discrimination increases aerial biomass and grain yield on rainfed bread wheat. Crop Sci. 42, 739-745.

Ritchie, J.T., 1981. Water dynamics in the soil-plant-atmosphere system. Plant Soil 58, 81-96.

Serraj, R., Krishnamurthy, L., Jyostna Devi, M., Reddy, M.J.V., Nigam, S.N., 2004a. Variation in transpiration efficiency and related traits in a groundnut mapping population. Int. Arachis Newslett. 24, 42-45.

Serraj, R., Krishnamurthy, L., Kashiwagi, J., Kumar, J., Chandra, S., Crouch, J.H., 2004b. Variation in root traits of chickpea (Cicer arietinum L.) grown under terminal drought. Field Crops Res. 88, 115-127.

Sheshshayee, M.S., Bindumadhava, H., Rachaputi, N.R., Prasad, T.G., Udayakumar, M., Wright, G.C., Nigam, S.N., 2006. Leaf chlorophyll concentration relates to transpiration efficiency in peanut. Ann. Appl. Biol. 148, 7-15.

Sinclair, T.R., Ludlow, M.M., 1986. Influence of soil water supply on the plant water balance of four tropical grain legumes. Aust. J. Plant Physiol. 13, 329-341.

Wright, G.C., Hubick, K.T., Farquhar, G.D., 1988. Discrimination in carbon isotopes of leaves correlates with water-use efficiency of field-grown peanut cultivars. Aust. J. Plant Physiol. 15, 815-825.

Wright, G.C., Hubick, K.T., Farquhar, G.D., Rao, R.C.N., 1993. Genetic and environmental variation in transpiration efficiency and its correlation with carbon isotope discrimination and specific leaf area in peanut. In: Ehleringer, J.R., Hall, A.E., Farquhar, G.D. (Eds.), Stable Isotopes and Plant Carbon/Water Relations. Academic Press, San Diego, pp. 247-267.

Wright, G.C., Nageswara Rao, R.C., Farquhar, G.D., 1994. Water-use efficiency and carbon isotope discrimination in peanut under water deficit conditions. Crop Sci. 34, 92-97. 